\title{
Smell impairment in patients with allergic rhinitis
}

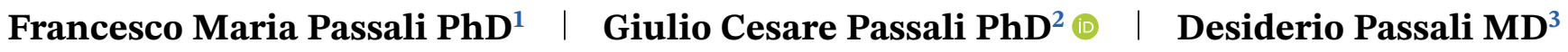 Giorgio Ciprandi $\mathrm{MD}^{4}$ (1)}

\author{
${ }^{1}$ Ear, Nose, Throat (ENT) Department, University Tor Vergata, Rome, Italy \\ ${ }^{2}$ ENT Department, University of Sacred Heart, Rome, Italy \\ ${ }^{3}$ International Federation Oto-Rhino-Laryngological (ORL) Societies (IFOS), Rome, Italy \\ ${ }^{4}$ Allergy Clinic, Casa di Cura Villa Montallegro, Genoa, Italy \\ Correspondence \\ Giorgio Ciprandi, MD, Allergy Clinic, Casa di Cura Villa Montallegro, Genoa 16132, Italy. \\ Email: gio.cip@libero.it
}

View this article online at wileyonlinelibrary.com.

\section{KEYWORDS}

allergic rhinitis, olfactometry, olfactory dysfunction, quality of life, real-life setting

Allergic rhinitis (AR) is a common disease characterized by type 2 inflammation. Allergic inflammation leads to AR's typical symptoms, including itching, sneezing, watery rhinorrhea, and obstruction. ${ }^{1}$ Allergic inflammation may also induce an olfactory dysfunction (OD), mainly through two pathogenic mechanisms: the nasal obstruction limiting the arrival of inspired air to the olfactory area, and direct damage of olfactory cells, including both neurons and structural cells. ${ }^{1}$ Moreover, an OD may be quantitative (anosmia or hyposmia) or qualitative (cacosmia). The measurement of OD may be subjective, as a self-reported symptom, or objective, using olfactometry.

However, few studies have investigated anosmia in the general population; the prevalence of self-reported anosmia ranged between $0.3 \%$ and $1.9 \%$ depending on different geographic areas. ${ }^{2-4}$ Consistently, OD is a neglected AR symptom rarely investigated in clinical studies.

Conversely, OD is an important symptom because it may suggest the presence of relevant allergic inflammation. In this regard, a recent systematic review evaluated the comprehensive literature concerning this issue, selecting 36 articles. $^{5}$ The reported OD prevalence in AR patients ranged between $20 \%$ and $40 \%$. The OD frequency increased with the AR duration and positively correlated with the AR severity. Consistently, an observational, crosssectional, and multicentre study (OLFAPEDRIAL) evaluated the sense of smell in 1260 children with AR. ${ }^{6}$ Forty- four percent of AR children reported smell dysfunction, with loss of smell frequency and intensity. Notably, the smell impairment correlated with disease severity and was more intense in persistent AR than intermittent AR. Guilemany et al. ${ }^{7}$ evaluated the sense of smell in 49 patients with persistent AR using Barcelona Smell Test-24 olfactometry. These authors observed a $67 \%$ prevalence of self-reported hyposmia. Moreover, there was a significant relationship between olfactory dysfunction, nasal obstruction, and type 2 inflammation. It also has to be underlined that smell impairment may affect AR patients' quality of life. ${ }^{8}$

Therefore, we investigated the prevalence of OD in a group of patients with AR enrolled in a real-world setting, such as a third-level rhinology clinic.

The study included 1063 AR patients (499 males, mean age 35.9 years) during the $2000-2019$ period. The inclusion criteria were adulthood (18-65 years) and documented AR diagnosis, based on the consistency between sensitization and symptom history, such as symptom appearance after exposure to the sensitizing allergen.

Exclusion criteria were chronic rhinosinusitis, asthma, recent upper airway infection, and comorbid diseases (e.g., head trauma, dementia, neurological disorders) or concomitant medications (e.g., corticosteroids, $\beta$-blockers, anti-thyroid drugs, dihydropyridine, angiotensin converting enzyme $[\mathrm{ACE}]$ inhibitors, and intranasal zinc) interfering with data interpretation. 
TA B L E 1 Prevalence and percentages of olfactory dysfunction and mean with SD of the TDI score in patients with allergic rhinitis $(\mathrm{N}=$ 1063)

\begin{tabular}{lcc} 
& Patient prevalence and frequency $\mathbf{n}$ & TDI (mean \pm SD) \\
Symptoms & $\mathbf{( \% )}$ & $10.4 \pm 4.4$ \\
\hline Anosmia & $160(15)$ & $22.2 \pm 3.9$ \\
Hyposmia & $308(29)$ & $30.2 \pm 4.1$ \\
Cacosmia & $42(4)$ & $32.6 \pm 3.5$ \\
Normosmia & $553(52)$ & \\
\hline
\end{tabular}

Abbreviations: SD, standard deviation; TDI, threshold discrimination identification.

The investigators used the Sniffin' Sticks olfactory test (Burghart Instruments, Wedel, Germany) to measure the threshold discrimination identification (TDI) score, as described. ${ }^{9}$ Patients were classified as normosmic (TDI $>30.5$ ), hyposmic (TDI $<30.5$ and $>16.5$ ), and anosmic (TDI $\leq 16.5$ ), whereas values $<5$ were not considered to be reliable. Cacosmia was evaluated as a self-reported complaint.

Table 1 reports the frequency distribution and TDI values of OD in the AR patients. Anosmia affected 160 (15\%) patients with mean TDI 10.4; 308 (29\%) patients had hyposmia with mean TDI 22.2; and 42 (4\%) patients reported cacosmia with normal TDI (mean 30.2). It has to be noted that cacosmia, as a qualitative defect, did not impair olfactometry.

Therefore, the current real-world study confirmed the relevant prevalence of OD in AR patients. Almost half of the patients presented with an OD. This relevant outcome underlined the clinical importance of smell impairment during AR. Namely, OD may have profound relevance for the patient with a remarkable impact on quality of life and may be dangerous up to life-threatening.

The present results were also consistent with the literature as they confirmed the high prevalence of OD in AR patients.

The current study has some strengths, including the large sample size and the objective measurement of OD. On the other hand, the main limitations include the lack of a complete evaluation of factors that could impact the TDI scores, including comorbidity and type of allergy, quality of life measurement, and biomarkers assay. Moreover, patients presenting to a rhinology clinic are likely biased by severity, and thus the current estimate may not be generalizable.

In conclusion, the present study demonstrated and confirmed that AR impacted olfactory function in about half of patients. Consequently, smell deserves careful attention in AR workup and management.

\section{CONFLICT OF INTEREST}

None.

\section{O R C I D}

Giulio Cesare Passali PhD (1) https://orcid.org/0000-00028176-0962

Giorgio Ciprandi MD (10 https://orcid.org/0000-0001-70168421

\section{RE F E RE N C ES}

1. Wise SK, Lin SY, Toskala E, et al. International consensus statement on allergy and rhinology: allergic rhinitis. Int Forum AllergyRhinol. 2018;8(2):108-352.

2. Hoffman HJ, Ishii EK, Macturk RH. Age-related changes in the prevalence of smell/taste problems among the United States adult population. Ann N Y Acad Sci. 1998;855:716-722.

3. Mullol J, Alobid I, Mariño-Sanchez F, et al. Furthering the understanding of olfaction, prevalence of loss of smell and risk factors: a population-based survey (OLFACAT study). BMJ Open. 2012;2: 001256.

4. Kang JW, Lee YC, Han K, Kim SW, Lee KH. Epidemiology of anosmia in South Korea: a nationwide population-based study. Sci Rep. 2020;10:3717.

5. Stuck BA, Hummel T. Olfaction in allergic rhinitis: a systematic review. J Allergy Clin Immunol. 2015;136:1460-1470.

6. Langdon C, Guilemany JM, Valls M, et al. Allergic rhinitis causes loss of smell in children: the OLFAPEDRIAL study. Pediatr Allergy Immunol. 2016;27(8):867-870.

7. Guilemany JM, García-Piñero A, Alobid I, et al. Persistent allergic rhinitis has a moderate impact on the sense of smell, depending on both nasal congestion and inflammation. Laryngoscope. 2009;119:233-238.

8. Passàli GC, Ralli M, Galli J, Calò L, Paludetti G. How relevant is the impairment of smell for the quality of life in allergic rhinitis?. Curr Opin Allergy Clin Immunol. 2008;8:238-242.

9. Hummel T, Kobal G, Gudziol H, Mackay-Sim A. Normative data for the "Sniffin' Sticks" including tests of odor identification, odor discrimination, and olfactory thresholds: an upgrade based on a group of more than 3,000 subjects. Eur Arch Otorhinolaryngol. 2007;264:237-243.

\section{How to cite this article:}

Passali FM, Passali GC, Passali D, Ciprandi G . Smell impairment in patients with allergic rhinitis. Int Forum Allergy Rhinol. 2021;1-2. https://doi.org/10.1002/alr.22786 